Negre, F., Marín, V.I. \& Pérez, A. (2013). Estrategias para la adquisición de la competencia informacional en la formación inicial de profesorado de primaria. Revista Electrónica Interuniversitaria de Formación del Profesorado, 16 (2), 1-12.

DOI: : http://dx.doi.org/10.6018/reifop.16.2.180751

\title{
Estrategias para la adquisición de la competencia informacional en la formación inicial de profesorado de primaria
}

Francisca Negre Bennasar, Victoria Irene Marín Juarros, Adolfina Pérez Garcies

Universitat de les Illes Balears (UIB)

\section{Resumen}

En la actual sociedad de la información y la comunicación una de las competencias requeridas para sus ciudadanos es la capacidad de gestionar grandes cantidades de información de una forma eficiente y adecuada a propósitos o intereses concretos. Por ello, el objetivo principal de esta experiencia es el diseño y evaluación de estrategias didácticas para la gestión de la información, concretamente en el contexto de la formación inicial de profesores de primaria. Los resultados de la experiencia avanzan en el reajuste de un modelo de curación de contenidos orientado hacia la adquisición de la competencia informacional.

\section{Palabras clave}

Formación de profesorado; Tecnologías de la Información y de la Comunicación; Gestión de la información; Enseñanza superior.

Contacto

Francisca Negre Bennasar, xisca.negre@uib.es, Departamento de Pedagogía Aplicada y Psicología de la Educación - Universitat de les Illes Balears (UIB). Cra. de Valldemossa, km 7.5. E-07122 Palma de Mallorca, España.

El presente trabajo forma parte del Proyecto de Investigación EDU2011-25499, que está desarrollando el Grupo de Tecnología Educativa (GTE) de la Universitat de les Illes Balears desde 2012, gracias a la financiación del Ministerio de Educación y Ciencia de España en el marco del Programa Nacional de Investigación Fundamental. 


\title{
Strategies for acquiring information competence in initial training of primary school teachers
}

\begin{abstract}
In today's information and communication society one of the skills required for its citizens is the ability to manage large amounts of information in an efficient way and appropriate to specific purposes or interests. Therefore, the main objective of this research is the design and development of didactical strategies for the management of information, particularly in the context of initial training of primary school teachers. The results of the experiment make progress in readjust a content curation model oriented towards the acquisition of the information competency.
\end{abstract}

\section{Key words}

Teacher training; Information and Communication Technologies; Information management; Higher education.

\section{Introducción}

En la sociedad de la información y el conocimiento el profesorado requiere gran capacidad para adaptarse a los cambios producidos, fundamentalmente, como consecuencia del proceso de tecnificación que caracteriza a la sociedad actual. Manejar y gestionar información es, cada vez más, un requerimiento derivado de esta transformación social, económica, cultural y, cómo no, educativa. El profesorado debe asumir su rol de formador y participar activamente en este proceso de cambio aceptando el compromiso de formarse de forma permanente.

El Grupo de Tecnología Educativa (GTE) de la UIB, con la intención de aportar mejoras en la formación del profesorado, está llevando a cabo el proyecto "EDU2011-25499: Estrategias metodológicas para la integración de entornos virtuales institucionales, sociales y personales de aprendizaje", en el que se desarrollan diferentes líneas de investigación orientadas al diseño de estrategias metodológicas para una adecuada explotación de los sistemas de gestión del conocimiento. Una de estas líneas se dirige al diseño y experimentación de estrategias didácticas que integren la red personal de conocimiento (gestión de la información y red de personas) como elemento del proceso formal de enseñanza-aprendizaje (E-A). Esta línea constituye el marco de acción de la experiencia que se presenta.

En este sentido, la gestión de la información o competencia informacional emerge como necesidad indispensable para poder desarrollar las funciones propias de un docente del siglo XXI y el profesorado de primaria tiene la responsabilidad de desarrollar esta competencia en el alumnado en las primeras etapas de su formación. La competencia informacional se manifiesta, por tanto, como un requerimiento para la formación que se debe ofrecer en los Centros de Educación de Infantil y Primaria (CEIP) desde la formación inicial en la Universidad.

Este artículo pretende seguir el trabajo iniciado en la asignatura "Gestión de proyectos TIC" del tercer curso de los estudios de Grado de Maestro de Primaria (UIB), optativa de la 
Mención de tecnología Educativa (Marín, Moreno y Negre, 2012), en relación a la búsqueda de estrategias didácticas para la adquisición de la competencia informacional.

\section{Planteamiento teórico}

Entre las funciones derivadas del nuevo rol que debe desarrollar el profesor, Torrego (2012) destaca el de organizador de la interacción entre los alumnos y los objetos de conocimiento, el de generador de interrogantes y guía de los procesos de búsqueda, análisis, selección, interpretación, síntesis y difusión de la información. La forma que propone es "estimulando permanentemente a los alumnos en la iniciativa y en el aprendizaje activo con creación, comunicación y participación" (Torrego, 2012, 129) y es la base sobre la que se fundamenta la metodología desarrollada en la asignatura que se presenta en este artículo.

Según Cassany y Ayala $(2008,68)$, “...estamos experimentando un cambio cultural profundo y relevante en las formas de usar, construir, hacer circular y divulgar el conocimiento". Por ello, la formación inicial del profesorado debe incluir estrategias didácticas dirigidas a trabajar y adquirir la necesaria competencia informacional ya que "... no se tiene el conocimiento necesario para aprovechar las ventajas que se pueden obtener mediante la utilización de las TIC" (Torrego, 2012, 130).

Estas estrategias didácticas deben acompañarse, por tanto, de las herramientas tecnológicas que posibiliten la iniciativa y el aprendizaje activo, la creación, la participación y la comunicación que promueve Torrego (2012). Onrubia (2007) ya avanzaba la importancia de las Tecnologías de la Información y la Comunicación (TIC) en la formación para promover actuaciones y formas de organización novedosas e inéditas: "los usos de las TIC más relevantes desde el punto de vista de la innovación docente universitaria han de ser, a nuestro juicio, usos de valor añadido, que transformen, en el sentido fuerte de la palabra, las formas en que profesores y estudiantes interactúan en el aula, y con ello sus formas de enseñar y aprender" (Onrubia, 2007, 23).

Como se comenta anteriormente, la competencia informacional engloba las "habilidades, conocimientos, disposiciones y conductas que capacitan a los individuos para reconocer cuándo necesitan información, dónde localizarla, cómo evaluarla y darle un uso adecuado de acuerdo con el problema que se les plantea" (Area, 2010, 52). Esta competencia está estrechamente relacionada con el proceso de curación de contenidos ("content curation"), que consiste en mantener, conservar y añadir valor a la información encontrada a través de su ciclo de vida (Digital Curation Centre, 2010). Por tanto, el curador de contenidos se encargaría de este proceso que implicaría la búsqueda, selección, almacenamiento, valoración, distribución y construcción de información. El ciclo de curación del que partimos es el que se presenta e implementa en la experiencia descrita en Marín, Moreno y Negre (2012) como resultado de la mejora del modelo anterior descrito en de Benito, Darder, Lizana, Marín, Moreno y Salinas (2013).

\section{Objetivo}

El objetivo principal de esta experiencia es proveer a los estudiantes del grado de primaria de estrategias didácticas para la adquisición de la competencia informacional mediante el diseño de un Entorno Virtual de Formación (EVEA) como actividad de aprendizaje y evaluación.

El resultado esperado a medio-largo plazo es formar a futuros docentes de primaria en temas relacionados con la competencia informacional para que puedan transferir el conocimiento en los centros de primaria desarrollando propuestas innovadoras a partir de la búsqueda y curación de información. 


\section{Metodología}

\section{Contexto}

La experiencia se ha desarrollado en la asignatura "Diseño y Gestión de Entornos Virtuales de Formación", optativa de la Mención de Tecnología Educativa de $4^{\circ}$ curso de Grado de Maestro de Primaria. En la experiencia participan todos los alumnos matriculados en la asignatura organizados en tres grupos de trabajo. La actividad de aprendizaje y evaluación de la asignatura consiste en el diseño de un entorno virtual que responde a las necesidades formativas del profesorado de tres CEIPs de Mallorca identificadas en la asignatura cursada con anterioridad ("Diseño de Proyectos TIC", optativa de Mención de 3 r curso) a partir de la metodología Aprendizaje/Servicio.

La metodología docente se basa en los principios antes expuestos por Torrego (2012): el rol del profesorado es el de orientador de la acción y potenciador de la interacción entre los alumnos con el grupo/clase, con su propio grupo de trabajo y con el resto de grupos de trabajo. Una parte fundamental para poder desarrollar la actividad se centra en la búsqueda de información para el desarrollo del marco teórico y el análisis de experiencias relevantes relacionadas con el tema de cada grupo. Para ello, el rol del profesorado se ha centrado en guiar a los alumnos en los procesos de búsqueda, análisis, selección, interpretación, síntesis y difusión de la información para poder ser aprovechada por el resto de compañeros de la asignatura (grupo clase y grupos de trabajo) actuando como "curadores" de la documentación localizada y compartida. Se estimula, por tanto y siguiendo a la misma autora, la creación (mediante el diseño de un EVEA), la comunicación entre los alumnos (compartiendo y curando contenidos) y la participación (colaborando en la búsqueda y selección de información con su propio grupo y con el resto de los grupos de trabajo).

En la experiencia desarrollada en la asignatura anterior, la herramienta escogida para la estrategia de curación de contenidos y el desarrollo de la competencia informacional fue Scoop.it, sin embargo, tras la valoración de los resultados, se decidió hacer un cambio en la herramienta, de cara a optimizar el proceso descrito. En la experiencia que presentamos se ha utilizado Mendeley (http://www.mendeley.com/) que, a pesar de no ser una herramienta propiamente de curación de contenidos sino más bien de gestión de referencias bibliográficas, cumple con los objetivos de la estrategia y proceso descrito.

\section{Procedimiento}

La metodología seguida en esta experiencia ha sido la establecida por el modelo de gestión de proyectos IPECC (Iniciar, Planificar, Ejecutar, Controlar y Cerrar), continuando con el proceso de investigación enmarcado en el proyecto I+D de diseño y desarrollo de iteración constante.

El procedimiento seguido se detalla a continuación:

- Fase inicial: Inicio del proyecto. Se articula la visión del proyecto, se establecen metas y se definen las expectativas y ámbito de aplicación. En nuestro caso, se establece la sub-investigación dentro del I+D EDU2011-25499 en relación al diseño y experimentación de estrategias didácticas que integren la red personal de conocimiento (gestión de la información y red de personas) como elemento del proceso formal de enseñanza-aprendizaje.

- Fase 1: Planificación. Se concreta el alcance, se organiza el equipo de trabajo, se identifican las tareas específicas y actividades a completar y se desarrolla un plan de proyecto y calendario. Se diseña la guía docente de la asignatura y, al empezar la asignatura, cada grupo comienza a trabajar en la planificación de su proyecto. 
- Fase 2: Ejecución del proyecto. Se van alcanzando las metas y al mismo tiempo se resuelven problemas y se va construyendo el proyecto. También se establece el control del proyecto: monitorizando los cambios del proyecto, haciendo correcciones y ajustando expectativas y metas, así como el calendario para resolver problemas. Durante esta fase se desarrolla un segundo ciclo de mejora iterativa hacia los objetivos desarrollando la experiencia descrita en este artículo, con cambios en la estrategia seguida de curación de la información (de un curador "experto" por grupo a todos curadores de grupo) así como en la herramienta (de Scoop.it a Mendeley); este último fue el aspecto que presentó mayores inconvenientes en la experiencia anterior (Marín, Moreno y Negre, 2012). Dentro de la ejecución del proyecto la experiencia de curación se desarrolló en dos etapas:

- Etapa 1: Familiarización con la herramienta. Se realizó previamente un taller de uso de Mendeley para los objetivos previstos de la experiencia y se organizó un foro de dudas en caso de necesitar soporte técnico.

- Etapa 2: Organización de grupos y del proceso de curación. Se organizó a cada uno de los 3 grupos de forma que cada integrante debía curar un mínimo de un documento de interés general y tres específicos para avanzar en el proyecto de grupo. Para ello, se crearon los 3 grupos públicos (ya que presenta limitaciones de miembros en el caso de grupos privados) en Mendeley y se pidió que todos los alumnos se unieran a los otros grupos, para poder compartir con ellos también documentación de interés. Al mismo tiempo, el equipo docente también se unió a cada uno de los tres grupos para poder realizar el seguimiento del trabajo de curación de documentación.

- Fase 3: Control del proyecto. En esta fase se realiza la lectura y análisis de documentación de interés para cada grupo de trabajo y se realiza el informe del proyecto a partir de diferentes sesiones de control en las que cada grupo expone sus avances, que son comentados y valorados por el resto de grupos y por la profesora de la asignatura. En estas sesiones de control se proponen acciones de mejora que cada grupo integra en su trabajo.

- Fase 4: Cierre y evaluación del proyecto. Se entrega el proyecto, se comunican los resultados, se realiza el informe y la evaluación del proyecto. En el margen de tiempo marcado, cada grupo debe hacer entrega de su proyecto definitivo y defenderlo públicamente. Cada proyecto es valorado y puntuado desde una triple perspectiva: autoevaluación de cada miembro del grupo, evaluación de cada participante de la asignatura y evaluación por parte de la profesora. Tras esta valoración de proyectos de alumnos se pasa a la evaluación de la experiencia de sub-investigación en relación a la gestión de información y red de personas, mencionada en la fase inicial y presentada en este trabajo.

\section{Técnicas/instrumentos de recogida de información}

Para realizar la evaluación de la experiencia, se utilizaron diferentes técnicas de recogida de información.

\section{- Entrevistas}

Las entrevistas se desarrollaron de forma grupal, con algunas preguntas individuales. Duraron 40 minutos cada una aproximadamente y se grabaron para posterior análisis. Las preguntas versaban sobre los siguientes aspectos: 
1. Uso de la herramienta de referencias bibliográficas y curación Mendeley con el grupo de trabajo.

2. Sugerencias y observaciones del grupo de trabajo.

3. Uso de la herramienta de referencias bibliográficas y curación Mendeley de forma individual.

4. Sugerencias y observaciones del trabajo individual.

- Observación

Se diseñó una plantilla para la valoración del proceso de curación seguido por los diferentes grupos y alumnos usando la herramienta escogida (Mendeley) para la gestión de la información.

Para ello, se registró una vez por semana los siguientes datos de cada uno de los documentos compartidos por cada alumno en Mendeley:

- Referencia del documento, es decir, la referencia bibliográfica identificando el título y los autores, especialmente.

- Tipo: Se indica el tipo de documento; por ejemplo, artículo de revista, informe, entrada de blog, libro, etc.

- Comentario: Se incluye el comentario o nota que ha dejado el alumno que lo ha compartido en el mismo documento, independientemente de que fuera vía web o versión de escritorio. En este comentario el alumno debía revisar el documento, valorándolo tras la lectura e preferentemente indicando también para qué parte del proyecto podría ser adecuado.

- I/E: Se indica si se trata de un documento localizado internamente a través de los dos documentos ofrecidos por usuarios en Mendeley o externamente, haciendo búsquedas localizadas o genéricas en buscadores, bases de datos, otras páginas, etc. Este aspecto también se valoraba en las entrevistas pues no siempre era claro si había sido buscado interna o externamente.

- Interacción: Se indica si existe interacción con los compañeros a través de compartir el documento. Por ejemplo, la valoración de "Me gusta".

- Valoraciones compañeros: Se incluye la valoración de otros compañeros en relación al mismo documento.

- Análisis de documentos

Una vez entregados los proyectos finales de la asignatura, se analizaron para revisar la inclusión de referencias bibliográficas de los documentos valorados en los trabajos, aspecto que ayudaría a evaluar la efectividad y utilidad real final del proceso de curación.

\section{Resultados}

Tras esta experiencia se pueden resaltar dos resultados principales:

- Formación inicial profesorado respecto a la competencia informacional

Por un lado, el cambio de herramienta (de Scoop.it a Mendeley) supuso una facilitación y mejora, prácticamente en todos los casos, en el desarrollo del proceso de curación y del trabajo colaborativo en cada uno de los grupos. La mayoría de alumnos valoró como muy positivo el uso de Mendeley para compartir y valorar/comentar documentos en grupos, 
aunque destacaron también la dificultad de encontrar documentación para visualizar/descargar en su interior. Se compartieron un total de 72 documentos entre los tres grupos, con una media de 5,5 por persona. El porcentaje de cada tipo de documentos compartidos se puede observar en la siguiente figura:

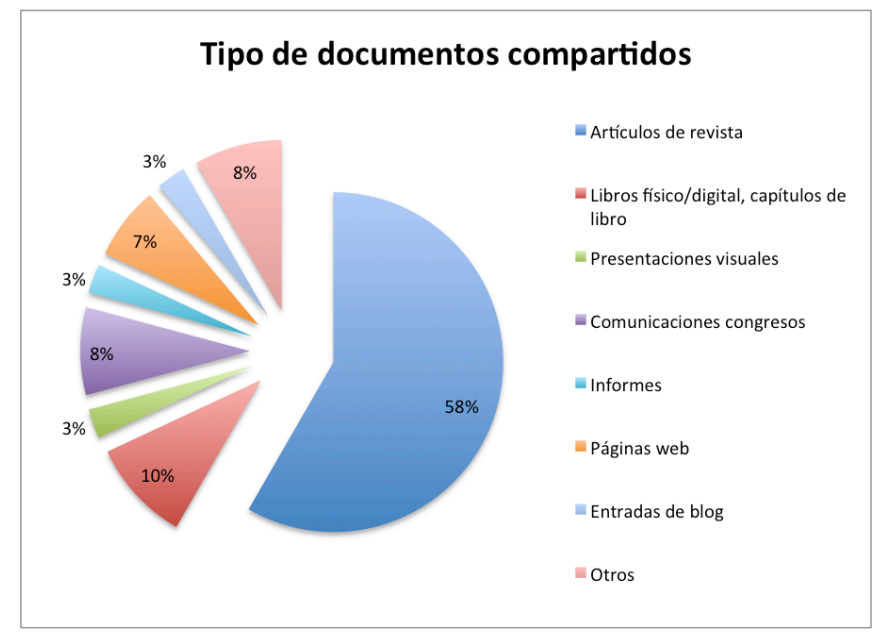

Figura 1. Tipo de documentos compartidos en Mendeley

En general, se destaca la dificultad de desarrollar estrategias para la búsqueda y selección efectiva de información, necesitando el establecimiento de palabras clave o sistemas para filtrar apropiadamente los documentos. También se destaca que es importante realizar el proceso de curación de forma periódica, de manera que se pueda ir revisando la documentación que se va compartiendo.

En cuanto al trabajo en grupo, la metodología utilizada también es valorada muy positivamente, especialmente el cambio realizado en la estrategia de curación. Como se ha mencionado antes, en la experiencia previa, la curación la realizaba todo el grupo, habiendo un curador "experto" responsable de moderar lo que compartía su grupo; este año todos los miembros de cada grupo tenían el rol de curadores sin haber "expertos", hecho que valoraron mejor pues así ninguno de ellos tenía la responsabilidad final del trabajo de filtrado grupal. En general, las búsquedas fueron externas a Mendeley, utilizando buscadores como Google y Google Académico y, en algunos casos, alguna base de datos como Dialnet.

Respecto al trabajo colaborativo en cada grupo, cada alumno que compartía con su grupo un documento, generalmente lo comentaba aportando su valoración e indicando, en algunos casos, en qué parte del proyecto podría encajar mejor, hecho que facilitó la tarea de redacción del marco teórico (todos los grupos incluyeron algunas referencias como resultado del proceso de curación en sus proyectos). Prácticamente en ningún caso se observa la aportación de valoraciones de los compañeros de grupo en esos documentos.

Por otro lado, se compartieron algunos documentos entre grupos, aunque en general no se percibieron de excesiva utilidad para cada proyecto, pues eran documentos genéricos que no se centraban en los aspectos concretos de que trataban cada uno de los proyectos.

Como tercer apunte, son pocos los alumnos que valoran el uso de Mendeley de forma individual, como gestor de referencias (utilidad principal de la herramienta), sino que más bien la entienden en contextos de trabajo de grupo. No se plantean seriamente utilizarla en 
un futuro cercano a nivel personal o profesional si no es para el trabajo colaborativo en desarrollo de proyectos. En este sentido, un $58 \%$ de los alumnos considera que sí utilizaría la herramienta en un futuro, mientras que un $25 \%$ indica que no y un $17 \%$ quizá.

Prácticamente ninguno de los alumnos ha utilizado Mendeley para otra actividad diversa a la propuesta en la asignatura (sólo un $8 \%$ la ha utilizado, para el proyecto final de grado). Sin embargo, la mayoría indica que no conoce herramientas con función similar (58\%). Los que dicen conocer otras herramientas citan Refworks (herramienta que se enseña en el primer año del grado) y Scoop.it (herramienta con la que trabajaron el pasado curso).

Respecto a las valoraciones de diferentes aspectos en la asignatura, se incluyen los siguientes apuntes, teniendo en cuenta la puntuación según el sistema de Likert de 1 a 4 , siendo 1 Totalmente en desacuerdo y 4 Totalmente de acuerdo:

a) Valoración de la importancia de la curación: 3,33.

b) Valoración de la herramienta empleada: 3.

C) Valoración de la estrategia de curación: 3,83.

d) Valoración de la metodología de la asignatura: 4.

e) Valoración de la importancia de la competencia en búsqueda de documentación: 3,92 .

f) Valoración de la importancia de la competencia en curación de documentación: 3,92 .

- Avance en el modelo de curación

Partimos del ciclo de curación resultado de la experiencia anterior expuesta en Marín, Moreno y Negre (2012) y hemos avanzado hacia el reajuste del modelo ampliando y modificando algunos aspectos:

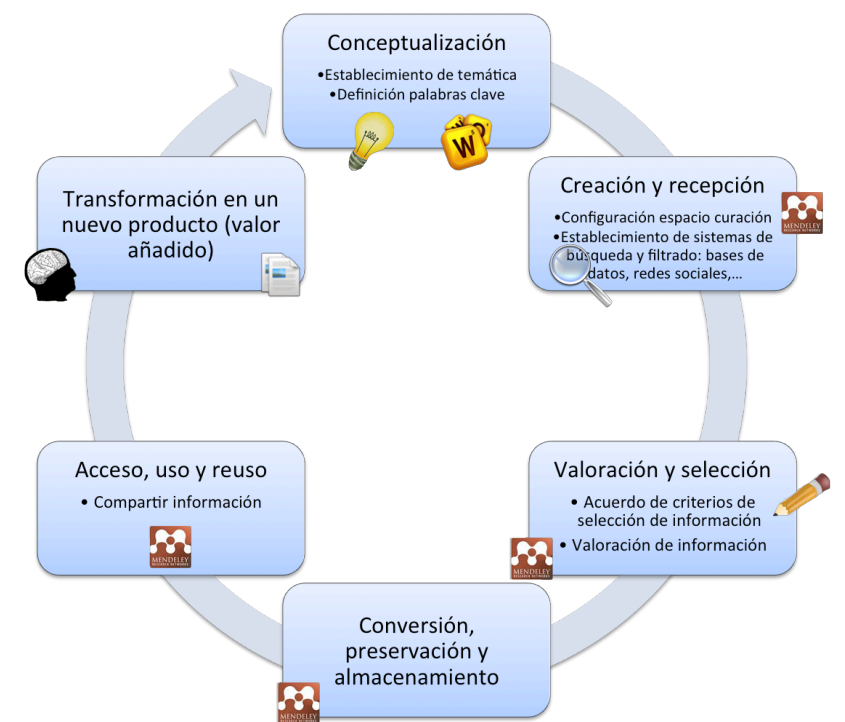

Figura 2. Ajuste del ciclo de curación en esta experiencia

En la primera fase de conceptualización, se hace mayor énfasis en el establecimiento de la temática para la cual se cura la información y, especialmente, en la definición de palabras 
clave ajustadas a dicha temática y que facilitarán la búsqueda y selección posterior de documentación.

En la siguiente fase, de creación y recepción, aparte de la configuración del espacio donde se efectuará la curación, es fundamental el establecimiento de sistemas de búsqueda y filtrado de información, tales como base de datos, redes sociales, buscadores, sistemas de curación, etc.

En la fase de valoración y selección de información se hace hincapié en el acuerdo en relación a los criterios de selección de documentación válida, así como la valoración de esta.

En la cuarta fase se presentan combinadas tres de las fases contempladas en de Benito et al. (2013) y que ya se habían reajustado en Marín, Moreno y Negre (2012): conversión, preservación y almacenamiento, haciendo referencia a la parte del proceso orientada a guardar la documentación con los metadatos necesarios para su acceso posterior.

La quinta fase se centra en el acceso, uso y reuso de la información valorada y almacenada anteriormente, así como en compartirla a través de diferentes medios.

La fase que cierra el ciclo e inicia de nuevo es la transformación de la información en nuevos productos, que aportan un valor añadido al corpus de información existente y a los conocimientos previos.

\section{Discusión y conclusiones}

\section{Discusión de resultados}

La Formación del Profesorado en la Sociedad de la Información y el Conocimiento debe incluir como elemento del proceso de E-A la adquisición de competencias, referidas al diseño y experimentación de estrategias didácticas para la gestión de la información y la red de personas. Como ya se ha comentado, la competencia informacional se consolida como una necesidad formativa del profesorado. La inclusión de este tipo de estrategias en los planes de estudio del Grado de Maestro de Primaria se dirige a dar respuesta a esta necesidad.

En este sentido, la asignatura "Diseño y Gestión de Entornos Virtuales de Aprendizaje" colabora en esta línea de investigación iniciada en el I+D EDU2011-25499 experimentado esta competencia a partir de las funciones que, según Torrego (2012), debe desarrollar el profesor. En la experiencia presentada, el rol del profesor se encarga de organizar la interacción entre los alumnos y los objetos de conocimiento en función de los interrogantes que surgen del propio proyecto que se desarrolla y para lo que deben documentarse de forma colaborativa. El rol docente desarrollado se basa, por tanto, en guiar el aprendizaje de la competencia informacional a partir del aprendizaje activo (metodología Aprendizaje/Servicio), estimulando la creatividad en el diseño de propuestas formativas en entornos virtuales, potenciando la comunicación y la participación colaborativa a través de las TIC entre el grupo de trabajo y el grupo clase.

El análisis de los resultados permite valorar de forma positiva la experiencia y considerarla como un avance en la formación del profesorado en temas relacionados con la competencia informacional, con lo cual el objetivo planteado en este artículo se cumple de forma satisfactoria.

En resumen, esta experiencia supone un avance para minimizar la brecha entre la formación del profesorado y las necesidades formativas en la sociedad actual dirigidas al uso de las TIC para la gestión de la información, preparando a los futuros docentes para que implementen 
nuevas formas de organizar a los estudiantes en el aula y en espacios virtuales, potenciando la interacción y el trabajo colaborativo.

\section{Conclusiones}

La información obtenida supone un nuevo avance en la construcción de un modelo de integración de la gestión de la información al permitir experimentar con un modelo obtenido en fases anteriores de la investigación (De Benito et al., 2013; Marín, Negre y Moreno, 2012). Asimismo, este avance ha permitido reajustar diferentes funciones de la estrategia de curación, de acuerdo con la metodología de la investigación en que se enmarca (basada en diseño y desarrollo) de ciclos de mejora constante.

Futuras propuestas deben incluir estrategias didácticas dirigidas a trabajar de forma más específica aspectos como la definición de palabras clave (fase de conceptualización), establecimiento de sistemas de búsqueda y filtrado (fase de creación y recepción) y el acuerdo de criterios de selección de la información (fase de valoración y selección).

Para completar el proceso iniciado, paralelamente al desarrollo de experiencias en los planes de formación de maestro (Grado de Primaria), sería conveniente realizar experiencias de colaboración con equipos docentes de diferentes Centros de Primaria. Este proceso permitiría ajustar las estrategias a las necesidades y requerimientos que plantea el alumnado de primaria. La valoración de este tipo de experiencias permitirá, por una parte, mejorar la oferta formativa del profesorado en formación, y, por otra, conocer las necesidades y las dificultades reales en las escuelas para trabajar la competencia informacional con el alumnado de primaria. Esta información permitirá un reajuste y mejora en el diseño y experimentación de estrategias didácticas, a la vez que supondría una clara muestra de transferencia de conocimiento desde la universidad a la escuela, y viceversa.

\section{Bibliografía}

Area, M. (2010). ¿Por qué formar en competencias informacionales y digitales en la educación superior? Competencias informacionales y digitales en educación superior. Revista de Universidad y Sociedad del Conocimiento (RUSC), Vol. 7, nº2. Extraído de http://www.uoc.edu/ojs/index.php/rusc/article/view/v7n2-area/v7n2-area

De Benito, B., Darder, A., Lizana, A., Marín, V. I., Moreno, J., \& Salinas, J. (2013). Agregación, filtrado y curación para la actualización docente. Píxel-Bit. Revista de Medios y Educación, 42, 157-169. Extraído de http://acdc.sav.us.es/pixelbit/images/stories/p42/12.pdf

Cassany, D., \& Ayala, G. (2008). Nativos e inmigrantes digitales en la escuela. CEE Participación educativa, (9), 53-71. Extraído de http://www.mecd.gob.es/revistacee/pdf/ng-ayala-gilmar.pdf

Digital Curation Centre (2010). What is digital curation? [página web]. Extraído de http://www.dcc.ac.uk/digital-curation/what-digital-curation

Marín, V. I., Moreno, J., \& Negre, F. (2012). Modelos educativos para la gestión de la información en educación superior: Una experiencia de curación de contenidos como estrategia metodológica en el aula universitaria. EDUTEC, Revista Electrónica de Tecnología Educativa, 42. Extraído de http://edutec.rediris.es/Revelec2/Revelec42/experiencia_curacion_contenidos_estra tegia_metodologica_aula_universitaria.html 
Onrubia, J. (2007). Las tecnologías de la información y la comunicación como instrumento de apoyo a la innovación de la docencia universitaria. Revista Interuniversitaria de Formación de Profesorado, 58 (21,1), 21-36. Extraído de http://www.aufop.com/aufop/revistas/indice/impresa/14

Santoveña, S. M. (2012). La formación permanente del profesorado en entornos virtuales de aprendizaje. Revista Electrónica Interuniversitaria de Formación de Profesorado, 43 (15, 4), 69-77. Extraído de http://www.aufop.com/aufop/revistas/arta/digital/171/1748

Torrego, A. (2012). La utilización de los blogs como recurso educativo en el área de Lengua Castellana y Literatura. Revista Interuniversitaria de Formación de Profesorado, 43 (15, 4), 127-137. Extraído de http://www.aufop.com/aufop/revistas/arta/digital/171/1754 


\section{Autores}

Francisca Negre Bennasar

Es Doctora en Ciencias de la Educación y profesora titular del Departamento de Pedagogía Aplicada y Psicología de la Educación de la Universidad de las Islas Baleares. Investigadora del Grupo de Tecnología Educativa de la UIB. Sus líneas de investigación y docencia son: la innovación docente con TIC; el diseño y gestión de entornos virtuales de formación; diseño de modelos para la integración de las TIC; y atención a la discapacidad con el apoyo de las TIC

Victoria I. Marín Juarros

Es Profesora Asociada del Departamento de Pedagogía Aplicada y Psicología de la Educación de la Universidad de las Islas Baleares y doctoranda en Tecnología Educativa. Investigadora del Grupo de Tecnología Educativa de la UIB. Sus líneas de investigación son: la integración de las TIC en los procesos de enseñanza aprendizaje; los entornos personales de aprendizaje; los modelos flexibles de enseñanza aprendizaje; el diseño y gestión de entornos virtuales de formación; y estrategias didácticas con TIC

Adolfina Pérez Garcias

Es Doctora en Ciencias de la Educación y profesora titular del Departamento de Pedagogía Aplicada y Psicología de la Educación de la Universidad de las Islas Baleares. Investigadora del Grupo de Tecnología Educativa de la UIB. Sus líneas de investigación y docencia son: la integración de las TIC en los procesos de enseñanza aprendizaje; el diseño de medios didácticos; la innovación docente con TIC; el diseño y gestión de entornos virtuales de formación; estrategias didácticas y tutoría con TIC 